\title{
Fuzzy PID Control of Lower Limb Exoskeleton for Elderly Mobility
}

\author{
GHASAQ AL REZAGE and M.O.TOKHI \\ Department of Automatic Control and \\ Systems Engineering \\ The University of Sheffield \\ Sheffield, UK \\ gsmousal@sheffield.ac.uk
}

\begin{abstract}
This paper presents investigations into the development of an assistive device for elderly mobility. An exoskeleton is designed as an assistive device, to enhance the lower extremity and provide supporting torque to augment the torque of hip and knee during the walking cycle. A humanoid and actuated exoskeleton frame are designed in solid works and then imported to Visual Nastran 4D virtual environment. Fuzzy PID Control algorithm uses to control the actuated exoskeleton. Simulated walking tasks are performed with a humanoid wearing the exoskeleton. It is assumed that the humanoid representing an elderly person, is able to provide $60 \%$ of the whole torque required to complete the gait cycle, and the assistive device providing $40 \%$ of the overall torque.
\end{abstract}

Keywords- lower extremity exoskeleton; fuzzy PID controller; assistive device.

\section{INTRODUCTION}

The ageing population is accelerating from many parts of the world. Many parts of the world have been witnessing a global aging population. Some statistics show that the birth rate in all regions has decreased. These days, there are more elderly people than before and due to reduced number of young people to take care of them. Thus, much research is needed to address the problems of aging. The United Nations reports that the number of old people above 60 was $9.2 \%$ in 1990 , in 2013 increased by $2.5 \%$ from 1990 and is estimated to rise further to $21.1 \%$ by 2050 . This means the absolute number of the elderly people will double in the next three decades, expecting it to be more than 2 billion by 2050. Nearly, about two thirds of the world's elderly people live in the developing countries. By 2050, nearly 8 in 10 of the world's older population will live in the less developed regions. The older persons aged 80 years and above were $14 \%$ of the old population in 2013, and estimated to grow to $19 \%$ in 2050 , that means there will be three times the number of people aged 80 years and over in the world [1]. Age can cause significant changes in mobility for the elderly. Walking efficiency will decrease because of the loss of muscle strength due to reduced motor neuron, muscle fiber and aerobic capacity [2]. Exoskeletons have emerged as assistive technology within the robotic sector. Significant numbers of researchers have engaged in

978-1-4673-8692-0/16/\$31.00 (C)2016 IEEE development of assistive devices for empowering or rehabilitation purpose. The aim is to augment reduced power of the joint and carry part of the body weight or use as aid and substitute the human limb [3], [4] . There has been a growing number of exoskeletons designed for rehabilitation. One of the widely used and studied devices in this field is Lokomat developed by the Swiss company Hocoma, and most widely adopted exoskeleton for rehabilitation around the world [4], [5]. It is driven by dc motor and ball screw. In addition the device has hip and knee joint for each leg. Force sensor was used to estimate the interaction force between the exoskeleton and lower limb. Another prominent assistive device is developed by Ikehara using a combination of worm gear and flexible shaft. Hybrid controlling used for both knee and ankle joints. The device aims to help the elderly people experiencing loss in their leg length, so are unable to walk in stable manner [6]. The Sogang University presented an exoskeleton device called Exoskeleton for Power Assistive (EXPOS) to help elderly people and patients in their motion. The aim of the work was to minimize the weight and volume of the exoskeleton to be more convenient and suitable for walking and sitting. The device consists of wearable exoskeleton and smart walker. The wearable device is less than $3 \mathrm{~kg}$ in weight, and applies the mechanically driven tendon driven and it has motion detection sensor. The walker carries most of the heavy weight of the motor and the battery, and servo motor control [7]. Tsukuba University and Cyberdyne in Japan have developed a Hybrid Assistive Limb (HAL-3), as a wearable robot designed for different functions. The main goal is to help the disorder and elderly people in their walking. DC actuator is used to generate proper torque for both hip and knee. Force sensor is used in the sole of the foot to measure reaction force, while EMG sensor is attached to skin surface of leg to estimate the muscle activity [8]. Wearable Walking Helper (WWH) is gravity compensation wearable exoskeleton developed in order to enhance the ambulate functions of disabled and elderly people [9]. The torque provided by WWH is to assist the locomotion of patient proportional to the torque calculated based on approximated human model [10]. Walking Power Assist Leg (WPAL) is another exoskeleton developed to provide an assistance to elderly people during walking. The system incorporates hybrid. 
Control based on joints angle, velocities and interaction force information. Dc servomotor selected as actuation for knee and hip joints [11]. The control methodology is key technology in the exoskeleton area. Literatures shows that many types of controllers have been used such as EMG, PID and intelligent algorithms [12].PID control is the widely used for its reliability and simplicity. However PID control may not be very efficient for exoskeleton systems because of the system is complex and nonlinear [13]. Fuzzy logic is an example of intelligent algorithms, which has good adaptabilities to the exoskeleton system with uncertain parameters. The work presented in this paper builds on previous work of the authors [14], which was based on conventional PID control. The current work develops a fuzzy PID control mechanism by combining the PID controller with fuzzy logic for tracking and control human movement with exoskeleton.

\section{SYSTEM DESCRIPTION}

The Visual Nastran (Vn4D) software developed by MSC Software Corporation, is used in this work for development and simulation of humanoid and exoskeleton models. The VN software is a virtual environment allowing design and development of 3D models and assemblies with actuators, motors and joints. The developed models can be linked to Matlab and actuated and controlled, thus allowing development and testing of various control mechanisms [15].

\section{A. The Humanoid Model}

The major function of humanoid model is to replicate human-like physical system in terms of segmental length, height and mass [16]. The human model segment parameters were built according to anthropometric data presented by Winter (2009) The humanoid developed in this work is based on a human body of $1.70 \mathrm{~m}$ in height $(\mathrm{H})$ and $75 \mathrm{~kg}$ in weight (M) as shown in Fig.1.

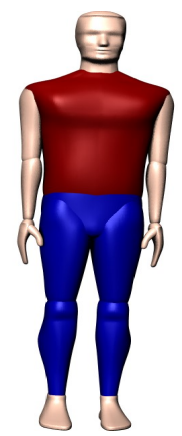

Figure 1: The Humanoid model

Vn4D software has the ability to connect the body segments to each other by joints provided known as constraints. The head and neck joints were considered as rigid joints. The shoulder, elbow, wrist and ankle joints are represented by revolute joints, while the knee and hip joints of right and left legs are represented by revolute motors in order to be controlled.

\section{B. Lower Limb Exoskeleton Dynamic}

The exoskeleton can perform many functions; the main purpose of this work is to provide assistive mobility for elderly. The second purpose is to provide assistive torque to augment the torque of the humanoid joint during the walking cycle. The mechanical design should be the first consideration such as Light weight and low cost material, in this project glass fiber reinforced polymer (GFRP) will be used due to its character and low cost. One of the goals of this project goal is to reduce the exoskeleton cost. The exoskeleton used in this work is expected to transfer the force directly to the ground therefore, the sole segment is included. The final model of exoskeleton is shown below in Fig.2. [17].



Figure 2: The Combination of Humanoid with Exoskeleton

The equation of motion of lower limb can be represented as:-

$$
M(\theta) \ddot{\theta}+C(\theta, \dot{\theta}) \dot{\theta}+G(\theta)=\tau
$$

where $\theta, \dot{\theta}$ and $\ddot{\theta}$ are the vector of the joint angle,angular velocity and acceleration, respectively. $M(\theta) \in R^{2 * 2}$ is the inertia matrix of the lower limb, $C(\theta, \dot{\theta}) \in R^{2 * 2}$ is the vector of centrifugal and Coriolis torque of human limb, $G(\theta) \in R^{2 * 2}$ is the vector of gravitational torque of the human limb, $\tau$ is the vector of actuator torque applied at the joint. the obtained $M(\theta)$ are as follows:

$$
\begin{gathered}
M_{11}=I_{1}+I_{2}+m_{1}\left(L_{c 1}\right)^{2}+m_{2}\left(L_{1}\right)^{2} \\
+m_{2}\left(L_{c 2}\right)^{2}+2 m_{2} L_{1} L_{c 2} \cos \left(\theta_{2}\right) \\
M_{12}=M_{21}=I_{2}+m_{2}\left(L_{c 2}\right)^{2}+m_{2} L_{1} L_{c 2} \cos \left(\theta_{2}\right) \\
M_{22}=I_{2}+m_{2}\left(L_{c 2}\right)^{2}
\end{gathered}
$$

The elements of $C(\theta, \dot{\theta})$ are represented as follow:

$$
\begin{gathered}
C_{11}=-m_{2} L_{1} L_{c 2} \sin \left(\theta_{2}\right) \dot{\theta_{2}} \\
C_{12}=-m_{2} L_{1} L_{c 2} \sin \left(\theta_{2}\right)\left(\dot{\theta_{1}}+\dot{\theta_{2}}\right) \\
C_{21}=m_{2} L_{1} L_{c 2} \sin \left(\theta_{2}\right) \dot{\theta_{1}} \\
C_{22}=0
\end{gathered}
$$


The parameter of the gravitational vector $G(\theta)$ are given by:

$$
\begin{gathered}
G_{1}=m_{1} L_{c 1} g \sin \left(\theta_{1}\right)+m_{2} g L_{1} \sin \left(\theta_{1}\right) \\
+m_{2} g L_{c 2} \sin \left(\theta_{1}+\theta_{2}\right) \\
G_{2}=m_{2} g L_{c 2} \cos \left(\theta_{1}+\theta_{2}\right)
\end{gathered}
$$

\section{Controller Design}

Conventional PID is a common controller used in controlling many of robotic systems. However, the limitation of PID algorithm is evident that it is adaptation to control the nonlinear and complex system; its parameters can not be tuned on-line. Therefore, a solution is introduced to deal with non-linearity and complexity, which existing the intelligent algorithm such as neural network and fuzzy theory [12].The intelligent algorithm has been widely used, and dose not need precise dynamic model [18]. Developing an adaptive controller by combining fuzzy logic with PID will integrate the advantages of both the fuzzy and PID also will reduce their disadvantages as illustrated from the previous work [13]. A fuzzy PID control mechanism is developed and used in this work.The structure of the fuzzy controller is shown in Fig.3. Two input and three output controller, the inputs are the error and the change of error, where the outputs are the modification of PID parameters $\Delta \mathrm{KP}, \Delta \mathrm{KI}$, and $\Delta \mathrm{KD}$.

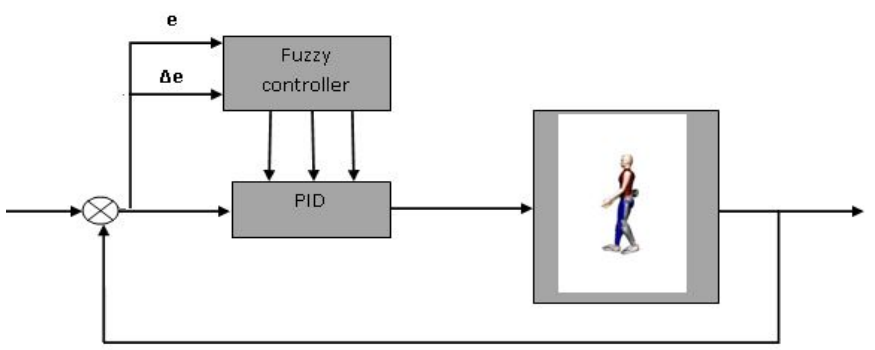

Figure 3: The Fuzzy/PID principle

Seven triangular membership functions were used leading to 49 fuzzy rules, these are Positive Big (PB),Positive Medium (PM), Positive Small(PS),Zero (Z),Negative Small (NS),Negative Medium (Nm), and Negative Big (NB) as shown in figure.4. The orientation of lower limb joints are independently controlled by Fuzzy/PID structure which have the similar rule bases.

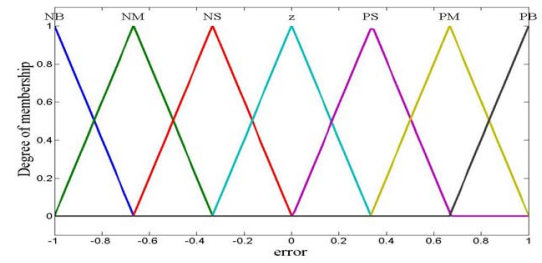

Figure 4: The Membership function

Four Fuzzy/PID controllers are developed in this work.The Fuzzy/PID control is designed in Matlab Simulink linked with VN4D environment.The control structure is shown in Fig.5.



Figure 5: The Controller structure

It is evident from reviewing the previous work, that the main issue of elderly mobility is body weight, so support should be provided by the exoskeleton system to the user all the time. The fundamental goal of the control method is to provide an assistive torque to enhance the ability of elderly people to walk. Many exoskeleton system designs do not take into consideration self-balancing control; instead, the user will control the exoskeleton balancing. Moreover, the measured real force generated by human muscle varies with different people. Depending on the CGA data, which is collected by recording video motion for standard walking cycle.The outputs of the controller are the torque of left hip(lh), left knee(lk), right hip(rh), and right knee(rk). These outputs will be used to control the joints hip and knee based on the references, which are sent to both the humanoid and the exoskeleton actuators in VN4D. $60 \%$ of power will drive humanoid, and the other $40 \%$ of the power will drive the exoskeleton as shown in Fig.6 [14] .

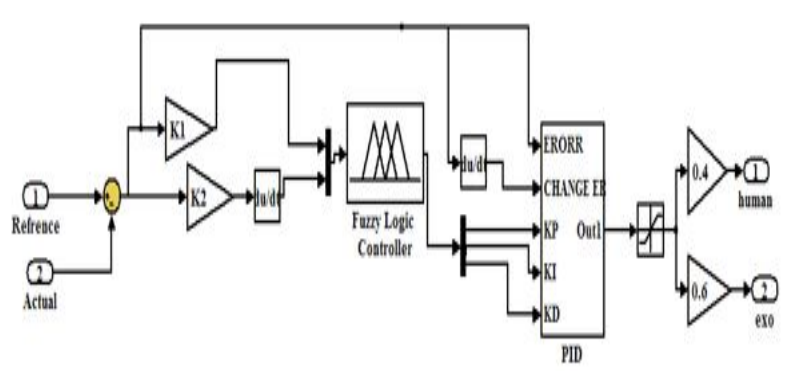

Figure 6: The Simulink block digram of fuzzy/PID

The adaptive fuzzy controller has two input and three output, the inputs of controller are error and change of error which there is $[1-, 1]$ while the outputs are respectively $\Delta \mathrm{kp}$, $\Delta \mathrm{ki}$, and $\Delta \mathrm{kd}$.For input the parameters $\mathrm{K} 1, \mathrm{~K} 2$ are tuned manually in order to get optimum performance. The fuzzy rules $\Delta \mathrm{KP}, \Delta \mathrm{KI}, \Delta \mathrm{KD}$ are shown in TableI, TableII, and TableIII. 
Table I: $\triangle$ KP Fuzzy Inference Rule

\begin{tabular}{cccccccc}
\hline$e / \dot{e}$ & NB & NM & NS & Z & PS & PM & PB \\
\hline NB & PB & PB & PM & PM & PS & Z & Z \\
NM & PB & PB & PM & PS & PS & Z & Z \\
NS & PM & PM & PM & PM & Z & NS & NS \\
Z & PM & PM & PM & Z & PS & NM & NM \\
PS & PS & PS & Z & NS & NS & NM & NM \\
PM & PS & Z & NS & NM & NM & NM & NB \\
PB & Z & Z & NM & NM & NM & NB & NB \\
\hline
\end{tabular}

Table II: $\Delta$ KI Fuzzy Inference Rule

\begin{tabular}{cccccccc}
\hline$e / \dot{e}$ & NB & NM & NS & Z & PS & PM & PB \\
\hline NB & NB & NB & NM & NM & NS & Z & Z \\
NM & NB & NB & NM & NS & NS & Z & Z \\
NS & NB & NM & NS & NS & Z & PS & PS \\
Z & NM & NM & NS & Z & PS & PM & PM \\
PS & NM & NS & Z & PS & PS & PM & PB \\
PM & Z & Z & PS & PS & PM & PB & PB \\
PB & Z & Z & PS & PM & PM & PB & PB \\
\hline
\end{tabular}

Table III: $\triangle K D$ Fuzzy Inference Rule

\begin{tabular}{cccccccc}
\hline$e / \dot{e}$ & NB & NM & NS & Z & PS & PM & PB \\
\hline NB & PS & NS & NB & NB & NB & NM & PS \\
NM & PS & NS & NB & NB & NB & NM & PS \\
NS & Z & NS & NM & NM & NS & NS & Z \\
Z & Z & NS & NS & NS & NS & NS & Z \\
PS & Z & Z & Z & Z & Z & Z & Z \\
PM & PB & NS & PS & PS & PS & PS & PB \\
PB & PB & PM & PM & PM & PS & PS & PB \\
\hline
\end{tabular}

\section{Simulations And Result}

The fuzzy PID control was implemented within MATLAB/SIMULINK, and this was linked with the humanoid and exoskeleton model developed in Vn4D software. The tracking references of knee and hip joints for left and right of each leg are shown in figures 7-10.



Figure 7: Hip joints reference and actual for left leg

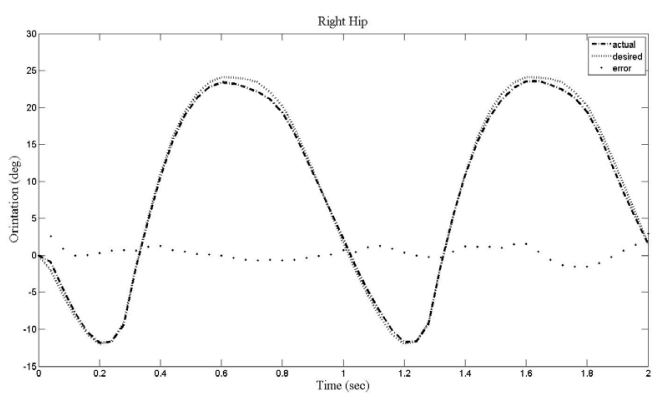

Figure 8: Hip joints reference and actual for right leg

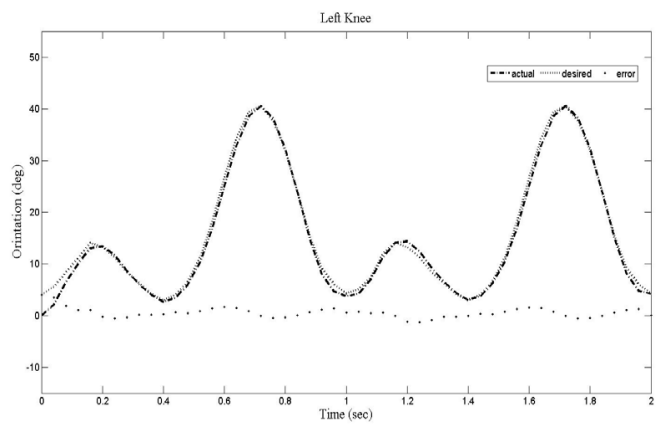

Figure 9: Knee joints reference and actual for left leg

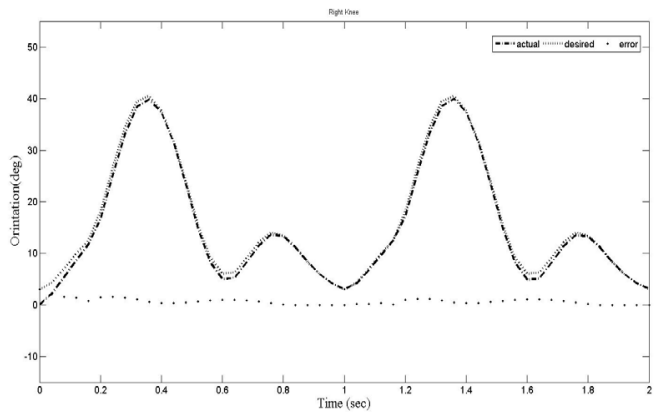

Figure 10: Knee joints reference and actual for right leg

The figures show that the Fuzzy PID controller successfully tracked the humanoid predefined references with low error between the desired and the measured trajectories. The green solid line is the reference trajectory, the blue solid line is the actual trajectory,and the red solid line is the error between the reference and the actual trajectory. It is evident that the system achieved better tracking as compared to just PID from the previous work [14].

The torque of humanoid for hip and knee joint measured during the walking cycle is illustrated in figures 11-14. Figure.11 shows the torque measured at the Hip joint of the humanoid for left and right leg, which was below 160 N.m through the walking cycle, however the knee torque for left and right leg was below $80 \mathrm{~N} . \mathrm{m}$ through the walking cycle as shown in figure. 12 . 


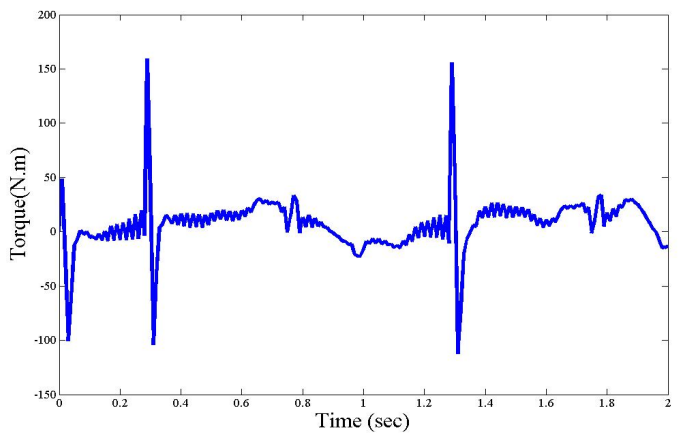

Figure 11: Hip torque profiles of humanoid during walking

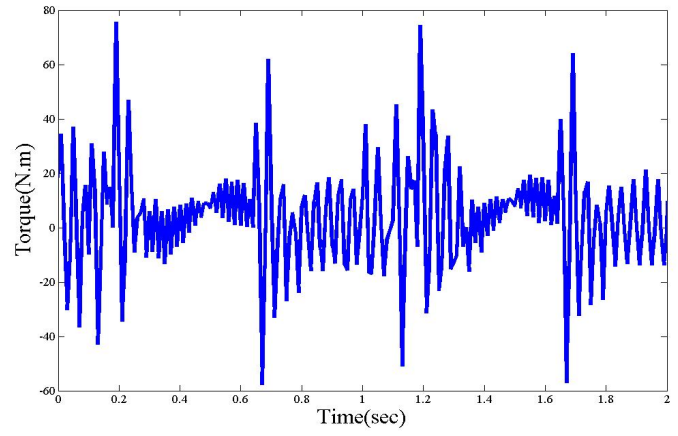

Figure 12: Knee torque profiles of humanoid during walking

Figures 13, 14 show the torque profile of the hip and knee joints, respectively for the humanoid and exoskeleton. It is evidence that the exoskeleton has provided about $40 \%$ of the total torque required for locomotion manoeuvre. The exoskeleton provided approximately $25 \mathrm{~N} . \mathrm{m}$ torque to enhance the knee joint and 60N.m torque to assist the hip joint , and these are all are acceptable according to Low [19],who reports that the maximum assistive torque to enhance the hip joint should be lower than 120 N.m , and for knee joint should be lower than 60 N.m.



Figure 13: Hip torque profiles of humanoid and exoskeleton during walking

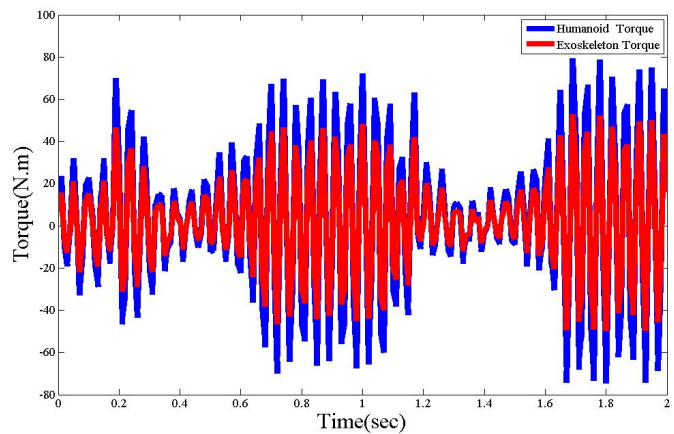

Figure 14: Knee torque profiles of humanoid and exoskeleton during walking

\section{CONClusion}

Humanoid model and actuated lower limbs exoskeleton device have been developed.Lower extremity exoskeleton has been developed to support the elderly mobility. A support torque $40 \%$ of the total torque required for the walking cycle is provided by the exoskeleton. This has been achieved with a Fuzzy PID controller for the knee and hip joints. The joint torque has been achieved within acceptable bounds. Future work will investigate sophisticated control algorithms such as type 2 fuzzy logic controller with an optimization algorithm for enhanced control performance.

\section{REFERENCES}

[1] U. Nations, "World population ageing 2013," Department of Economic and Social Affairs PD, 2013.

[2] F. Prince, H. Corriveau, R. Hébert, and D. A. Winter, "Gait in the elderly," Gait \& Posture, vol. 5, no. 2, pp. 128-135, 1997.

[3] J. L. Pons, Wearable robots: biomechatronic exoskeletons. John Wiley \& Sons, 2008.

[4] Y. Wei Hong, Y.-J. King et al., "Lower extremity exoskeleton: Review and challenges surrounding the technology and its role in rehabilitation of lower limbs," Australian Journal of Basic and Applied Sciences, vol. 7, no. 7, pp. 520-524, 2013.

[5] G. Colombo, M. Joerg, R. Schreier, V. Dietz et al., "Treadmill training of paraplegic patients using a robotic orthosis," Journal of rehabilitation research and development, vol. 37, no. 6, pp. 693-700, 2000.

[6] T. Ikehara, K. Nagamura, T. Ushida, E. Tanaka, S. Saegusa, S. Kojima, and L. Yuge, "Development of closed-fitting-type walking assistance device for legs and evaluation of muscle activity," in Rehabilitation Robotics (ICORR), 2011 IEEE International Conference on. IEEE, 2011, pp. 1-7.

[7] K. Kong and D. Jeon, "Design and control of an exoskeleton for the elderly and patients," Mechatronics, IEEE/ASME Transactions on, vol. 11, no. 4, pp. 428-432, 2006.

[8] H. Kawamoto and Y. Sankai, "Power assist system hal-3 for gait disorder person," Computers helping people with special needs, pp. 19-29, 2002.

[9] T. Nakamura, K. Saito, Z. Wang, and K. Kosuge, "Realizing modelbased wearable antigravity muscles support with dynamics terms," in Intelligent Robots and Systems, 2005.(IROS 2005). 2005 IEEE/RSJ International Conference on. IEEE, 2005, pp. 2694-2699.

[10] T. Nakamura, K. Saito, and K. Kosuge, "Control of wearable walking support system based on human-model and grf," in Robotics and Automation, 2005. ICRA 2005. Proceedings of the 2005 IEEE International Conference on. IEEE, 2005, pp. 4394-4399.

[11] F. Chen, Y. Yu, Y. Ge, and Y. Fang, "Wpal for human power assist during walking using dynamic equation," in Mechatronics and Automation, 2009. ICMA 2009. International Conference on. IEEE, 2009, pp. 10391043. 
[12] D. Pan, F. Gao, Y. Miao, and R. Cao, "Co-simulation research of a novel exoskeleton-human robot system on humanoid gaits with fuzzypid/pid algorithms," Advances in Engineering Software, vol. 79, pp. 3646, 2015.

[13] J. Niu, Q. Song, and X. Wang, "Fuzzy pid control for passive lower extremity exoskeleton in swing phase," in Electronics Information and Emergency Communication (ICEIEC), 2013 IEEE 4th International Conference on. IEEE, 2013, pp. 185-188.

[14] G. AL REZAGE, M. TOKHI, and S. K. ALI, "Design and control of exoskeleton for elderly mobility," in ASSISTIVE ROBOTICS: Proceedings of the 18th International Conference on CLAWAR 2015. World Scientific, 2016, pp. 67-74.

[15] S. Wang, "Software review: Motion simulation with working model $2 d$ and msc visual nastran 4d," Working Model 2D--Reviews and Awards, 2007.

[16] D. A. Winter, Biomechanics and motor control of human movement. John Wiley \& Sons, 2009.

[17] K. Lochan and B. Roy, "Control of two-link 2-dof robot manipulator using fuzzy logic techniques: A review," in Proceedings of Fourth International Conference on Soft Computing for Problem Solving. Springer, 2015, pp. 499-511.

[18] P. Shi, C. Lei, Y. Zhang, Y. Wang, and F. Wang, "Pid control of the mechanical legs based on fuzzy adaptive," in Cyber Technology in Automation, Control, and Intelligent Systems (CYBER), 2015 IEEE International Conference on. IEEE, 2015, pp. 1965-1970.

[19] K. Low, "Robot-assisted gait rehabilitation: From exoskeletons to gait systems," in Defense Science Research Conference and Expo (DSR), 2011. IEEE, 2011, pp. 1-10. 